\title{
A NEW CHARACTERIZATION OF UNICHORD-FREE GRAPHS
}

\author{
Terry A. MCKeE \\ Department of Mathematics and Statistics \\ Wright State University \\ Dayton, Ohio 45435 USA \\ e-mail: terry.mckee@wright.edu
}

\begin{abstract}
Unichord-free graphs are defined as having no cycle with a unique chord. They have appeared in several papers recently and are also characterized by minimal separators always inducing edgeless subgraphs (in contrast to characterizing chordal graphs by minimal separators always inducing complete subgraphs). A new characterization of unichord-free graphs corresponds to a suitable reformulation of the standard simplicial vertex characterization of chordal graphs.
\end{abstract}

Keywords: chordal graph, unichord-free graph, minimal separator.

2010 Mathematics Subject Classification: 05C75, 05C69, 05C15.

\section{REFERENCES}

[1] A. Berry, J.-P. Bordat and O. Cogis, Generating all the minimal separators of a graph, Internat. J. Found. Comput. Sci. 11 (2000) 397-403. doi:10.1142/S0129054100000211

[2] A. Brandstädt, F.F. Dragan, V.B. Le and T. Szymczak, On stable cutsets in graphs, Discrete Appl. Math. 105 (2000) 39-50.

doi:10.1016/S0166-218X(00)00197-9

[3] A. Brandstädt, V.B. Le, and J.P. Spinrad, Graph Classes: A Survey (Society for Industrial and Applied Mathematics, Philadelphia, 1999). doi:10.1137/1.9780898719796

[4] S. Klein and C.M.H. de Figueiredo, The NP-completeness of multi-partite cutset testing, Congr. Numer. 119 (1996) 217-222.

[5] T. Kloks and D. Kratsch, Listing all minimal separators of a graph, SIAM J. Comput. 27 (1998) 605-613. doi:10.1137/S009753979427087X 
[6] R.C.S. Machado and C.M.H. de Figueiredo, Total chromatic number of unichordfree graphs, Discrete Appl. Math. 159 (2011) 1851-1864.

doi:10.1016/j.dam.2011.03.024

[7] R.C.S. Machado, C.M.H. de Figueiredo and N. Trotignon, Complexity of colouring problems restricted to unichord-free and $\{$ square, unichord $\}$-free graphs, Discrete Appl. Math. 164 (2014) 191-199. doi:10.1016/j.dam.2012.02.016

[8] R.C.S. Machado, C.M.H. de Figueiredo and K. Vušković, Chromatic index of graphs with no cycle with unique chord, Theoret. Comput. Sci. 411 (2010) 1221-1234. doi:10.1016/j.tcs.2009.12.018

[9] T.A. McKee, Independent separator graphs, Util. Math. 73 (2007) 217-224.

[10] T.A. McKee, When all minimal vertex separators induce complete or edgeless subgraphs, Discrete Math. Algorithms Appl. 5 (2013) \#1350015. doi:10.1142/S1793830913500158

[11] T.A. McKee and F.R. McMorris, Topics in Intersection Graph Theory (Society for Industrial and Applied Mathematics, Philadelphia, 1999). doi:10.1137/1.9780898719802

[12] Y. Shibata, On the tree representation of chordal graphs, J. Graph Theory 12 (1988) 421-428. doi:10.1002/jgt.3190120313

[13] R.E. Tarjan, Decomposition by clique separators, Discrete Math. 55 (1985) 221-232. doi:10.1016/0012-365X(85)90051-2

[14] N. Trotignon and K. Vušković, A structure theorem for graphs with no cycle with a unique chord and its consequences, J. Graph Theory 63 (2010) 31-67. doi:10.1002/jgt.20405

[15] S.H. Whitesides, An algorithm for finding clique cut-sets, Inform. Process. Lett. 12 (1981) $31-32$. doi:10.1016/0020-0190(81)90072-7

Received 19 August 2014

Revised 2 March 2015

Accepted 2 March 2015 